\title{
Smart Blood Bag Management System in a Hospital Environment
}

\author{
Soo-Jung Kim ${ }^{1,3}$, Sun K. Yoo ${ }^{2,3}$, Hyun-Ok Kim ${ }^{4}$, Ha-Suk Bae ${ }^{5}$, Jung-Jin Park ${ }^{6,7}$, \\ Kuk-Jin Seo ${ }^{1,9}$, Byung-Chul Chang ${ }^{8}$ \\ ${ }^{1}$ Graduate School of Biomedical Engineering, Yonsei Univ. 134 Shinchon-dong \\ Seodaemun-ku Seoul, Korea \\ ${ }^{2}$ Dept. of Medical Engineering, Yonsei Univ. College of Medicine, 134 Shinchon-dong \\ Seodaemun-ku, Seoul, Korea, Correspondence \\ sunkyoo@yumc.yonsei.ac.kr \\ ${ }^{3}$ Center for Emergency Medical Informatics, 134 Shinchon-dong Seodaemun-ku, \\ Seoul, Korea \\ ${ }^{4}$ Dept. of Laboratory Medicine, Yonsei Univ. College of Medicine, 134 Shinchon-dong \\ Seodaemun-ku, Seoul, Korea \\ ${ }^{5}$ Dept. of Rehabilitation Medicine, Ewha Womans Univ. College of Medicine, 911-1 \\ Mok-dong Yangchun-ku, Seoul, Korea \\ ${ }^{6}$ Graduate School of Information, Yonsei Univ. 134 Shinchon-dong Seodaemun-ku Seoul, \\ Korea \\ ${ }^{7}$ Center for Signal Processing Research, Yonsei Univ. 134 Shinchon-dong, Seodaemun-ku, \\ Seoul, Korea \\ ${ }^{8}$ Dept. of Thoracic \& Cardiovascular Surgery, Yonsei Univ. College of Medicine, 134 \\ Shinchon-dong Seodaemun-ku, Seoul, Korea \\ ${ }^{9}$ Human Identification Research Center, Yonsei Univ. 134 Shinchon-dong Seodaemun-ku, \\ Seoul, Korea
}

\begin{abstract}
In order to provide suitable blood transfusion samples to patients, the blood bag should be kept at a uniformly maintained temperature to prevent deterioration during transportation. Therefore, this paper presents a blood monitoring and management system for use in hospitals. This system may continuously report the temperature of the blood bank refrigerator, track the location of a blood bag to increase staff operation efficiency, and can confirm that the assigned blood bag was transported to the intended patient in need of transfusion. We developed and demonstrated the clinical usability of the combined blood temperature management and tracking system using a ubiquitous sensor network and RFID (Radio Frequency Identification) technology.
\end{abstract}

Keywords: RFID, Sensor network, Location tracking system, Blood.

\section{Introduction}

For the development of ubiquitous healthcare, the use of RFID (Radio Frequency Identification) technology and Sensor Network is becoming more commonplace but there is still little research on the simultaneous use of these two systems together as one application. These systems can be used to greatly improve the tracking of blood used in transfusions, as the blood must match the intended patient and should be 
tracked from the time it leaves the blood bank to the time it arrives for use in transfusions, in order to ensure that the blood was handled according to regulations $[1,2]$. Blood should be kept at a fixed temperature. Blood that has deteriorated during transportation or while in storage must not be supplied to the patient. According to the Britain's National Blood Service report in 2000, $4.59 \%$ of the blood in stored blood banks of their hospitals was lost, and this percentage of lost blood may be greater in Korea's hospitals [3]. Therefore, there is a great need to construct a system using the Location Tracking Service and the sensor network that hospitals can use to measure blood temperature during both storage and transportation of blood bags, and to confirm that good-quality blood is supplied to the correct patients.

The blood bank currently operates the entire system manually, where the medical staff delivers blood to the location of transfusion after taking it out of refrigeration storage. The reasons why blood is not used for transfusions are because it was too old or there was an inadequate supply, or because the hospital administration denied use of the sample. Blood is always discarded if improper storage methods were used and by an increase in temperature resulted. Approximately thirty minutes after whole blood is removed from a $4{ }^{\circ} \mathrm{C}$ refrigerator and left to sit at room temperature, the red corpuscle becomes hemolytic; this decreases the lifetime of the blood because it causes excessive condensation and metabolism [1]. Thus, it is very important to keep blood at a temperature of $2 \sim 6{ }^{\circ} \mathrm{C}$ in order to maintain its quality and stability. Also, blood used for transfusions should be assigned to the correct patient. Accidents have been reported where the medical staff have inappropriately labeled the blood bags, mixed inappropriate blood samples together, or performed inaccurate adaptability tests [4].

We developed a 3T (Time, Temperature, Tracking)-enhanced system to prevent patient-blood mismatching and to obtain better temperature management of the blood samples by using a sensor network in blood banks and a RFID sensor tag that is placed directly on the blood bags. This system can track the movement of blood bags in designated time intervals (Time) and can monitor the change in blood temperature (Temperature) to provide more efficient, higher quality, and correct blood transfusions. The system can also generate data reports that medical personnel can share. Moreover, the need for an automatic system for transporting blood bags will help to reduce both the time and effort of medical staff. For these reasons, we used the Location Tracking System to track the location of blood bags and to prevent deterioration of blood that is being transported.

\section{RFID and Sensor Network Using Zigbee}

RFID is used to discriminate between objects that have a unique RF-tag, and this allows information to be processed specifically for each labeled object. RFID consists of a RF-tag, a RF-tag reader and operation software. The RF-tag reader can recognize the tag in 0.01 0.1 second and thus used in real-time application. The recognition rate is more than $99.9 \%$ in an area of $0 \sim 5 \mathrm{~m}$, making it possible to communicate in a fullduplex mode, which saves a maximum of $64 \mathrm{Kbyte}$ of data [5]. The RFID can use 
various ISM public frequency bands between $125 \mathrm{KHz}$ and $2.45 \mathrm{GHz}$. Presently, the high frequency band RFID of $13.56 \mathrm{MHz}$ is practically used in traffic cards in Korea because of the low tag cost compared to that of the low frequency band. The RFID is also divided into passive and active tags according to battery usage. The active RFID tag has a battery and can attach to various sensors.

The IEEE 802 standardization group defines the protocol standard according to various transmission rates and distances, such as the 802.11 WLAN and the 802.15.1 Bluetooth [6]. Among these standards, Zigbee, which is based on 802.15.4 for the MAC and PHY layer protocols, is frequently used in monitoring and controlling applications that are low power, low rate, and low cost for wireless networks. Zigbee uses the $2.45 \mathrm{GHz}$ ISM band and accesses the channel by the CSMA-CA method. Zigbee is useful for sensor networks because it can extend to a wide area, being able to connect to 255 devices within a network, transmit data at a maximum of $250 \mathrm{Kbps}$, and support mesh network configurations [7].

\section{Materials and Methods}

\subsection{Hardware}

\section{(1) Sensor, RFID tag \& read/writer}

We used the Crossbow Technology MTS420CA sensor to record the temperature of the refrigerator, the blood bank room temperature, and the temperature of the blood bag. The MTS420CA is used with the MICAz mote, which was developed by UC Berkeley to monitor the temperature, humidity, light acceleration, etc. The IEEE 802.15.4 is used to transmit the temperature data to the sink node, which is a MIB510CA which is also attached to the MICAz platform mote. Each sensor node is powered by two AA batteries and the signal from these sensors can reach $20 \sim 30 \mathrm{~m}$ distance but they can only operate for 10 15 hours in high power consumption mode, which does not have any programmed sleep intervals [8].

For the RFID temperature sensor, tag, and tag reader, we used the TempSens from KSW Co., which is an active RFID tag using an ISM band of 13.56 MHz. This has a built-in paper type battery that can last over 16 months and has enough SRAM memory to save 64 measurements of temperature data (Table 1) [9].

Table 1. Sensor, RFID tag and read/writer hardware specification

\begin{tabular}{|c|c|c|}
\hline & Sensor \& RF module & RFID tag \& read/writer \\
\hline Model & $\begin{array}{c}\text { Crossbow Technology } \\
\text { MTS420CA, MPR2400, MIB510CA }\end{array}$ & KSW TempSens, Inside \\
\hline Frequency & $2.4 \mathrm{GHz}$ ISM band & $13.56 \mathrm{MHz}$ ISM band \\
\hline Standard & IEEE 802.15 .4 & ISO 15693-3 \\
\hline Interface & RS-232C & USB \\
\hline Data rate & $250 \mathrm{Kbps}(57600 \mathrm{bps}$ baud rate) & Upper $115200 \mathrm{bps}$ baud rate \\
\hline Operation Temperature & $40 \sim 123.8^{\circ} \mathrm{C}$ & $-15 \sim+50^{\circ} \mathrm{C}(\mathrm{tag}), 20 \sim 70{ }^{\circ} \mathrm{C}(\mathrm{read} / \mathrm{writer})$ \\
\hline Etc & $\pm 0.5^{\circ} \mathrm{C}$ at $25^{\circ} \mathrm{C}$ accuracy & $\begin{array}{c}\text { Data memory for } 3 \mathrm{methods} \\
(\text { all, threshold, max } / \mathrm{min})\end{array}$ \\
\hline
\end{tabular}




\section{(2) Location Tracking System}

The Location Tracking System is frequently used to send and receive small items using the container shown in Fig. 1. This system consists of a host computer, container, station, operator, controller, shift controller, and other components [10]. The host computer completely manages the entire system, and the station component sends for and receives the container. The operator loads the container and then sends the container to the final destination by having the container follow the tracks assigned by the controller and the shift controller.

In this paper, we used the Auto Track System from the SFA Co., which has been developed to be compatible with Siemens's equipment. The host computer met the following specifications of being a Pentium $4 \mathrm{CPU}$ with $2.53 \mathrm{GHz}, 512 \mathrm{MB}$ of RAM, and $40 \mathrm{~GB}$ of HDD.

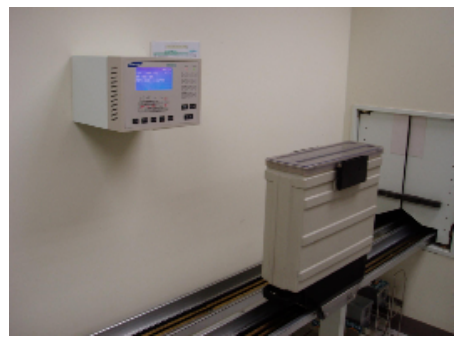

Fig. 1. Container, station and operator of the Auto Track System

\section{(3) Others}

We used the IBM ThinkPad R40 as the laptop computer for the blood bank which was attached to the sink node and the RFID reader. The HP TC1100 and the HP iPAQ RW6100 were used for the tablet PC and the PDA (Personal Digital Assistants), respectively. The DB Server was a Pentium $4 \mathrm{PC}$ which was a CPU with $2.8 \mathrm{GHz}$, $200 \mathrm{~GB}$ of HDD, 1 GB of RAM, and was embodied with a MSSQL Server2000.

\subsection{System Configuration and Data Gathering}

The whole system configuration, the data acquisition process, and the configuration of the Location Tracking System from the blood bank to the place of transfusion are described in Fig. 2. The process begins with the refrigerator located inside the blood bank. In it is a sensor that configures the sensor network using Zigbee RF communication with the outside sink node. The data is then simultaneously sent to both the laptop computer and the RFID reader. Both the temperature and the blood bag information are stored in the DB Server, which is available through the WLAN within the hospital and is also connected to the HIS (Hospital Information System). This allows medical staff to access the information via the web server within the WLAN limits.

When the blood is taken from the blood bank refrigerator, the Location Tracking System transports the blood from the blood bank's station to the designated final destination. At this time, the host computer and controller determine the container's 
track and alert the medical staff to the arrival of the containers. The host computer shows where the container is located at any given time, allowing medical staff to track the blood and decide whether or not to send it back depending on their readiness to receive it. Also, the host computer can be accessed by the DB Server, which allows users to query the blood location and the time the blood was released from the blood bank by using their monitoring devices.

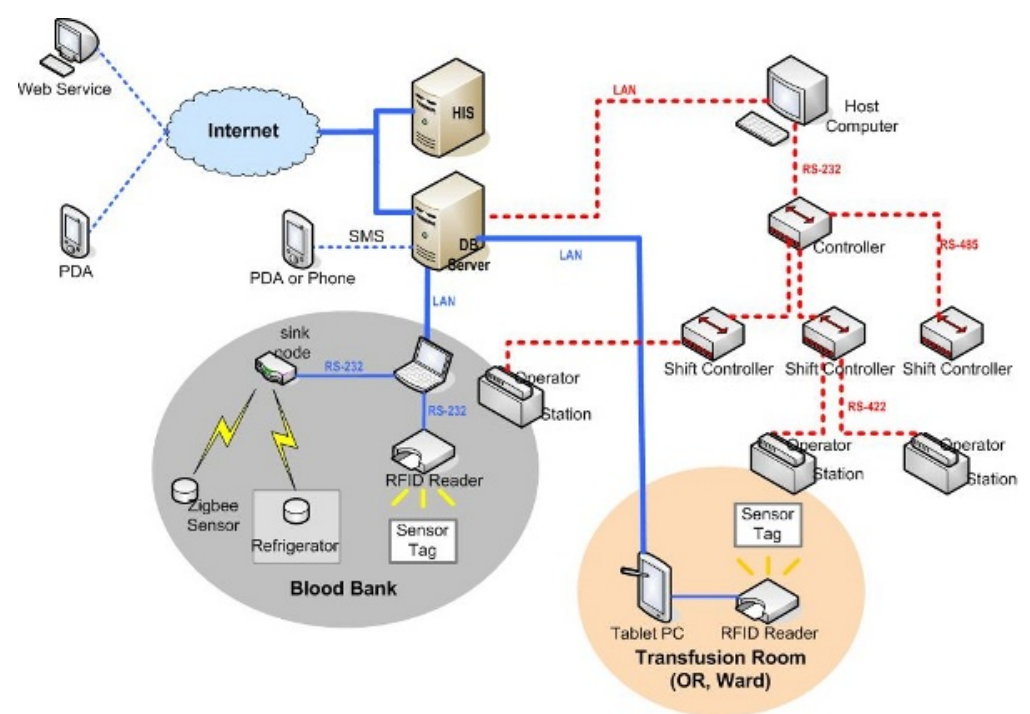

Fig. 2. Combined configuration of the temperature monitoring and the location tracking system

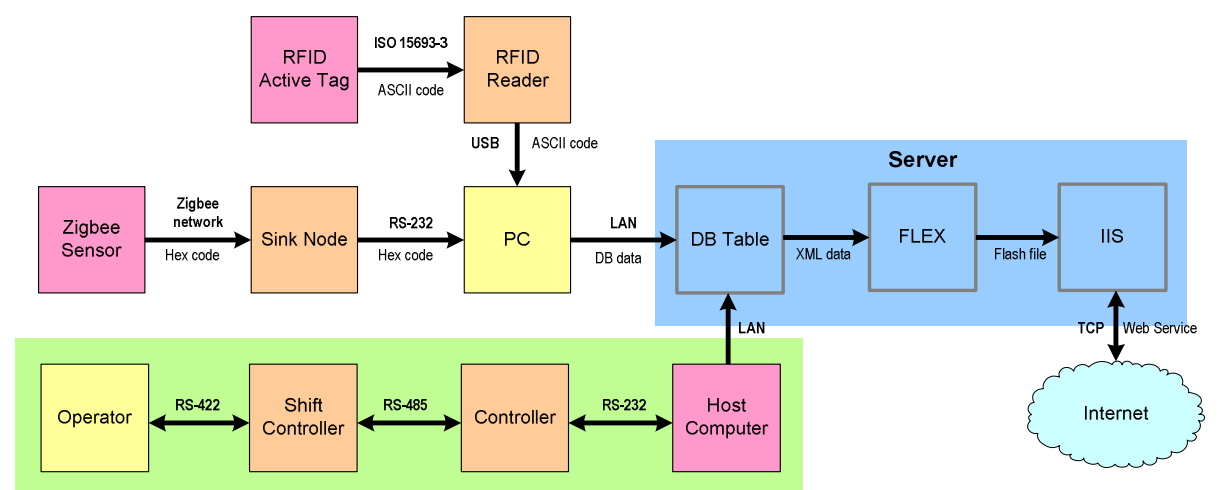

Fig. 3. Data gathering flow chart

A more concrete flow of data acquisition for the system described above is presented in Fig. 3. The cluster of the blood band is divided into two kinds of connections which are centered on the laptop PC. The laptop PC acquires the raw 
data, as a hex code for the Zigbee sensor network and as an ASCII code for the RFID tag reading, from each of these connections. Thereafter, the server receives the data from the laptop to compose the DB table. At this time, the information on the location and time the blood was sent from the blood bank is saved in the DB. The DB Server converts this real-time information into XML script and the FLEX program then converts it into Flash form, enabling users in remote places to access the information via the web using Internet Explorer. The programs used in the sensor network, RFID reading, and the PDA application are realized with Microsoft Visual $\mathrm{C}++6.0$, C\#.NET, and .NET Compact Framework, respectively.

\section{Results}

\subsection{Blood Transfusion Process}

To verify the usability and efficiency of the system described above, we tested the system in the Shin-chon Severance Hospital located in Seoul, Korea. To prevent indirect deterioration of blood, the sensor network using the Zigbee mote and the temperature sensor installed in the blood bank refrigerator were used. This sensor measures the temperature inside the refrigerator and transmits measurements to the sink node and the laptop, which is connected to the DB server through WLAN within the hospital. If the temperature inside the refrigerator were to reach a temperature not suitable for blood storage, the DB server would send a SMS message to alert the administrator of the problem. When donated blood first arrives at the blood bank, the

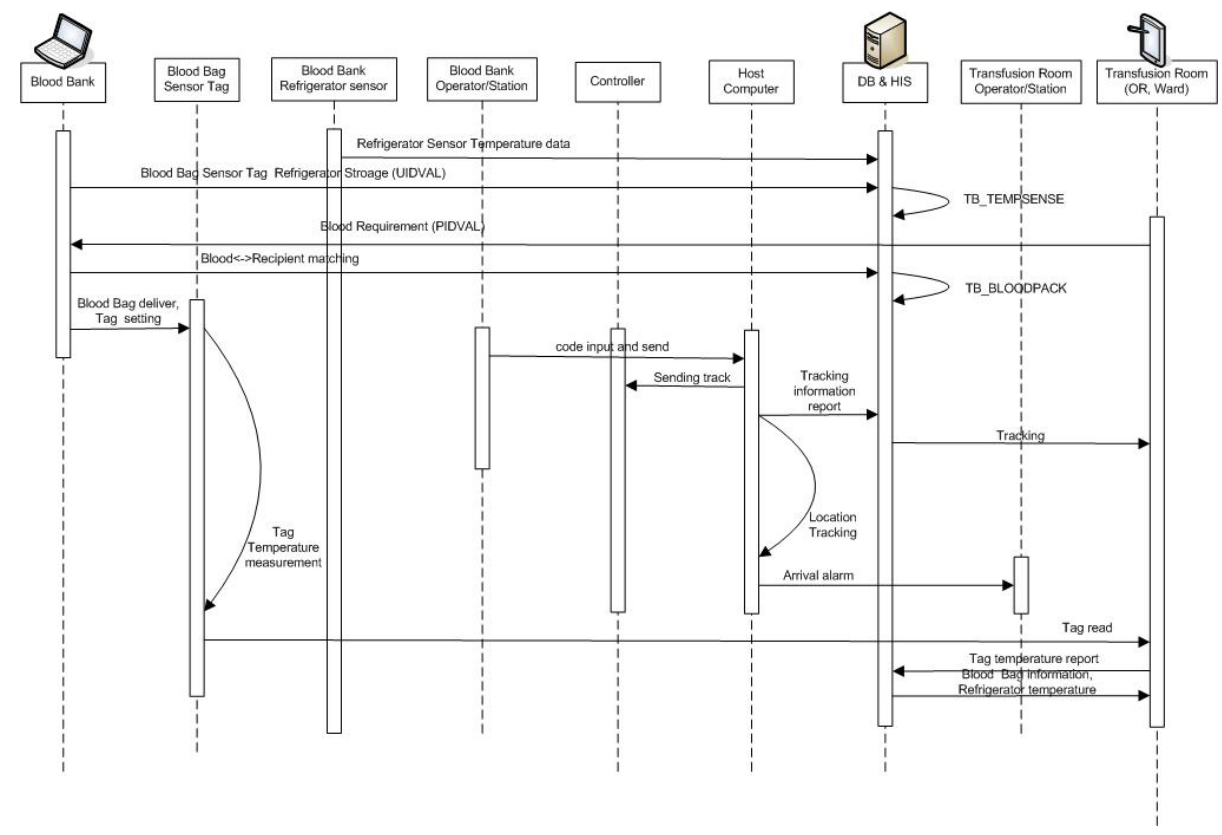

Fig. 4. Sequence diagram starting from the blood donation to the blood transfusion 
medical staff instantly labels the blood bags with unique RFID tags and stores them in the refrigerator until needed. When blood is requested for a transfusion, the blood bank saves the blood bag information collected during storage and programs the tag to measure the temperature of the blood bag until it is received by the medical staff waiting to receive the sample. As the blood bag is transported to the final destination station via the Location Tracking System track, the host computer estimates the container's location at every station en route, and records this information in the DB server. Therefore, the remote administrator or the medical staff waiting at the final destination can know where the blood bag is at any given time. The host computer can also inform the destination station of the blood container's estimated time of arrival. Upon arrival, the destination station reports the temperature of the storage refrigerator prior to delivery and the temperatures of the blood bag during delivery, as measured by the RFID tag. The temperature of the blood is the most important environmental information to deciding whether the blood is suitable for use in a transfusion (Fig. 4).

We measured the temperature of the blood bank refrigerator in five-minute increments, because temperature does not fluctuate significantly within seconds in a controlled environment. Although the storage temperature is continuously monitored, the RFID tag on the blood bag not only contains this information but also the duration of storage time, and provides the blood bag with a unique ID. When blood is requested, the blood bank programs the RFID sensor to measure the temperature in pre-determined time intervals. A used RFID tag can only save 64 measurements of temperature data. Assuming it would take approximately an hour to transport the blood, we programmed the tag to measure the temperature in one-minute time intervals (Fig. 5).

As shown in Fig. 6, the blood is moved through the Location Tracking System. The host computer monitors all moving containers and provides their location, temperature, and the time the sample left the blood bank (Fig. 6 (d)).

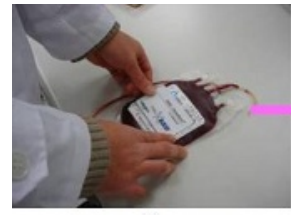

(a)

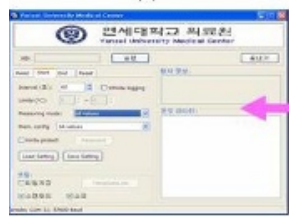

$(f)$

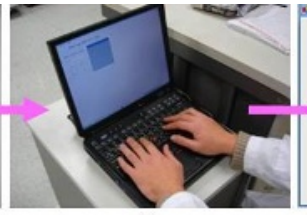

(b)

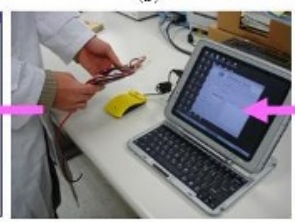

(e)

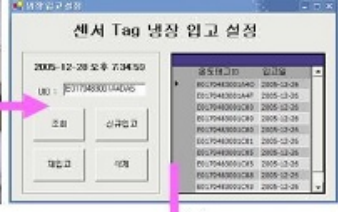

(c)

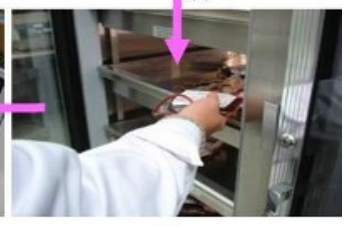

(d)

Fig. 5. Storage/take-out process of blood: (a) Attaching the RFID tag on the blood bag, (b) Storage set up, (c) Storage software, (d) Storage, (e) Tag setting after removal, and (f) Software used for tag setting 


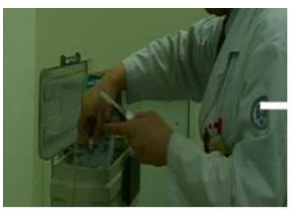

(a)

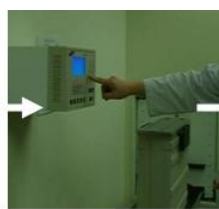

(b)

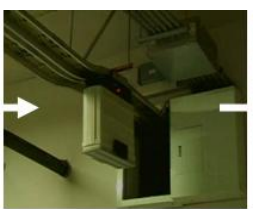

(c)

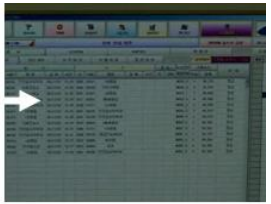

(d)

Fig. 6. Transport of blood using the Location Tracking System: (a) Input of the blood bag into the container, (b) Programming the destination, (c) Container moving along the track, and (d) Screenshot of the host computer tracking all containers

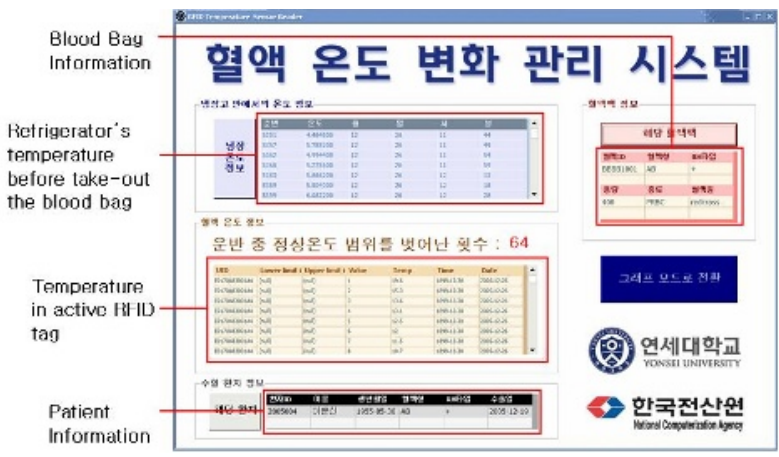

Fig. 7. Management system reporting the variation in temperature of the blood bag before a transfusion

Medical staffs always verify that the temperature of blood meets blood handling regulations, and that the intended patient and the delivered blood match, before the transfusion is performed (Fig. 7). When the RFID reader reads the tag, the temperature information during transport is first shown in the tablet PC. The storage temperature of the refrigerator in the blood bank also helps the medical staff determine whether the blood should be used for transfusion.

A remote administrator can use the DB data to query the blood temperature and patient information, to track deterioration of blood quality, or to confirm that a sample was used in a previous transfusion, as illustrated in Fig. 8. The information of both the blood bag and the refrigerator can be displayed in the same window as graphs, and a table can be generated to include the RFID tag ID, temperature and time at PDA.

To examine the possibility of using the Blood Management and the Location Tracking System as a real application, we tested the error range of the temperature sensor tag. The reader can recognize a RFID tag on the blood bag when held within 3 $\mathrm{cm}$ of the reader. If two or more tags are placed within this recognition range, the reader will not read any of the tags. Five sensor tags were used to compare the measured temperature to that of a digital thermometer $\left(5^{\circ} \mathrm{C}\right)$. This resulted in an insignificant difference of $0.5 \sim 1{ }^{\circ} \mathrm{C}$. 


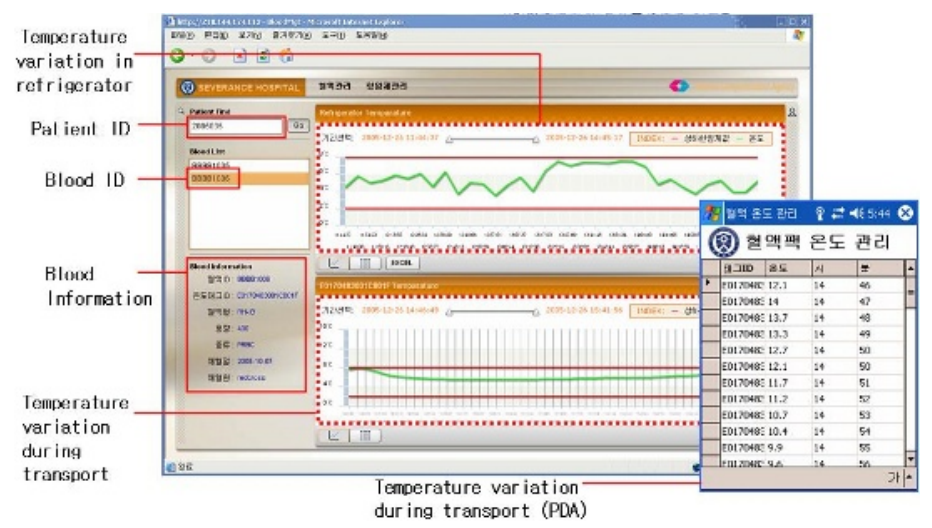

Fig. 8. Difference in temperature of a blood bag as displayed on Internet Explorer

Figure 9 represents the results of sensor 5's temperature rise an hour after the blood bag was removed from the refrigerator for transfusion. The reason this sensor tag consistently reported the highest temperature is because the blood bag with this sensor was placed on top of the other bags and therefore had the most contact with room temperature air. The graph indicates that this blood can no longer be used for transfusion because it exceeded $8^{\circ} \mathrm{C}$ after 45 minutes, and suggests that the bag could have been used within 30 minutes after removal from the refrigerator because a temperature of $6^{\circ} \mathrm{C}$ is still within regulations for blood transfusion.

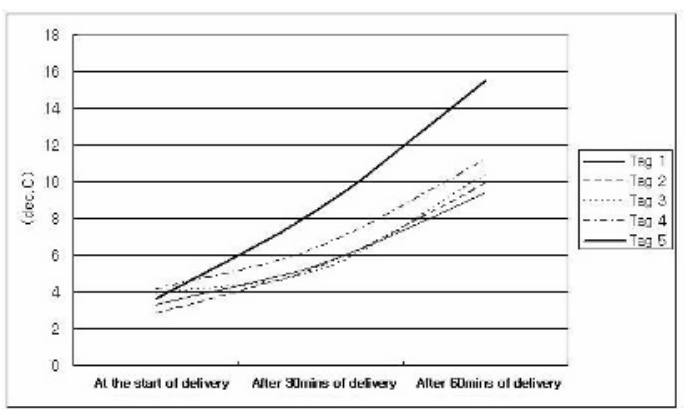

Fig. 9. Changes in temperature of the blood bags after removing them from the refrigerator

The time it takes to send a blood bag by the Location Tracking System can directly affect the rise in temperature as shown in Fig. 10. Most requests for blood were made during the normal working hours over the course of a 24-hour period. Deliveries took an average of 8 9 minutes. They took only 7 8 minutes except in cases where an operation was rescheduled and the previously arrived container was sent back. Moreover, excluding the $3.7 \%$ of containers sent back more than five times due to reoperation, the remaining containers do not take more than 15 minutes to be sent (Table 2). As Fig. 11 illustrates, often times the containers from the previous day are 
sent to the storage station at the blood bank around 5:00 A.M. This increased the average time it took for a sample to reach its destination. Therefore, our system can be used as a reasonable way to automate the transportation of blood. This system will deliver blood in a timely manner, as long as medical staff realizes the possible back$\log$ that could occur at 5:00 A.M.

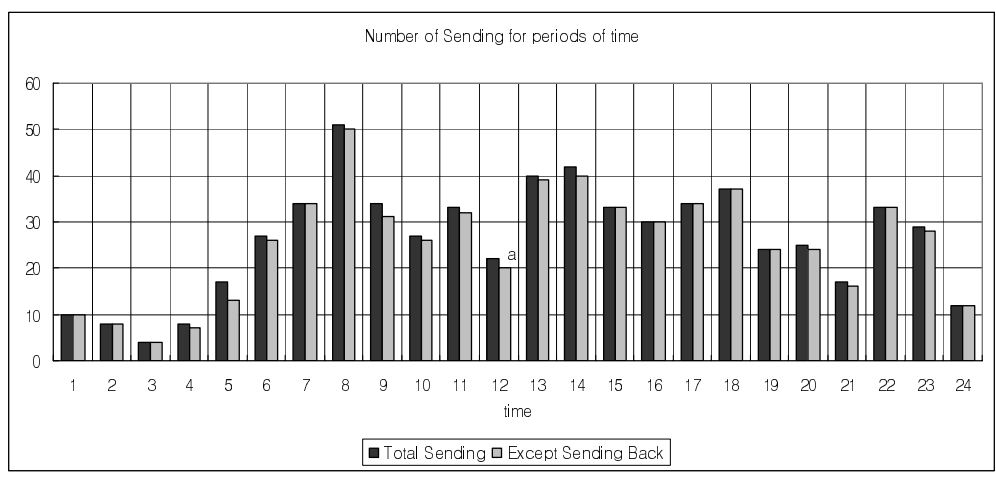

Fig. 10. Number of containers sent by the blood bank over a 24-hour time period

Table 2. Sending rate and average time of sending

\begin{tabular}{|c|c|}
\hline $\begin{array}{c}\text { Sending rate of blood bank among the all sending } \\
\text { results in hospital (\%) }\end{array}$ & 16.04781 \\
\hline Average time of sending (min) & 8.2884 \\
\hline Probability of re-operation (\%) & 5.07 \\
\hline Excessive probability of re-operation (\%) & 3.17 \\
\hline $\begin{array}{c}\text { Average time of sending except excessive probability } \\
\text { of re-operation (min) }\end{array}$ & 7.6514 \\
\hline
\end{tabular}

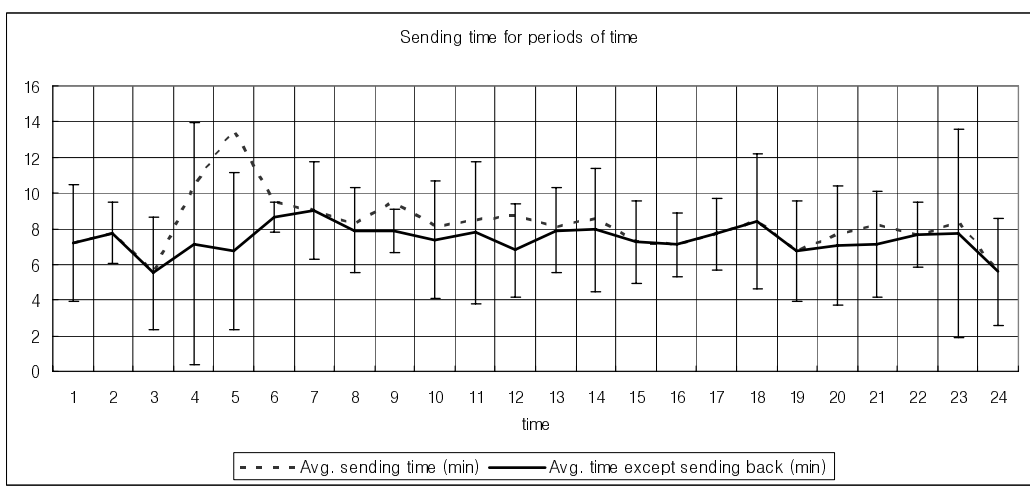

Fig. 11. Sending time and variance of containers sent from blood bank over a 24-hour period of time 


\section{Conclusions and Discussion}

The hospital blood bank is in charge of managing blood bags from the time they are received from the blood donor to the time they are sent to the blood recipients for transfusion. Blood is very sensitive to temperature and should be stored in a temperature-stable environment, such as a refrigerator, to prevent deterioration during storage and transportation. Before the blood transfusion can take place, a number of tests are first performed by the medical staff to determine whether the blood is suitable for transfusion and to confirm the temperature of blood bag. However, the blood bank's storage facilities and equipment are still susceptible to power supply failures and human error, as they manually report the temperature every four hours [11]. Therefore in this paper, the blood bank sensor network system and the RFID tag for use during blood transport were developed to be more effective than manpower alone, while having the ability to share data and provide correct provisions of blood. The Location Tracking System proved useful, as we were able to track the location of moving blood bags and the time required of the medical staff was reduced, freeing them to do other necessary tasks.

Our test of the system proved that a more correct and analytical data history can be obtained than with the existing fragmentary management system, and we made it easy to monitor the real-time temperature variance of the blood samples. Based on this systematic blood management system, it is possible to decrease the rate of human error, the negative side effects of using poor quality blood for transfusions, and the rate of blood that is discarded. While our system makes managing blood bags simple and reliable, it also has a few technological shortcomings. The RFID tag which has a built-in sensor and battery is still too expensive to introduce to most medical centers, as it seems to be technically verified by a reliable organization. Also, the lifetime of the sensor battery is too short to be practical and must be co-developed to use with both the sensor network and the RFID. Finally, it is necessary to develop more graphical PDA programs to increment the mobility of the tag reader. It would be ideal if the container could be controlled thermostatically to preserve the temperature of blood bags throughout the transportation process.

\section{Acknowledgement}

This study was supported by a grant of the Korea Health $21 \mathrm{R}$ and D Project, Ministry of Health and Welfare, Republic of Korea (02-PJ3-PG6-EV08-0001) implemented A Study on Experiments of Management of Blood Temperature with RFID Temp. Sensor \& Management of Anticancer Medicine with Wireless Sensor Node, NCA (National Computerization Agency)'s USN Field Test. .

\section{References}

[1] Blood Center, the Republic of Korea National Red Cross. http://www.bloodinfo.net/

[2] Ministry of Health and Welfare. http://www.mohw.go.kr/index.jsp

[3] The National Blood Service (2000). http://www.blood.co.uk/ 
[4] Using RFID Technologies to Reduce Blood Transfusion Error, Joseph Dalton, PhD, White Paper by Intel Corporation, Cisco Systems, San RAaffaele Hospital (2005)

[5] Fundamentals and Applications in Contactless Smart Cards and Identification, G. Lee, YoungJin.com (2004)

[6] Zigbee Alliance Tutorial, Bob Heile, Zigbee Alliance (2005)

[7] Zigbee Network Layer Overview, Ian Marsden, Zigbee Alliance (2005)

[8] Crossbow Technology, Inc. http://www.xbow.com/

[9] KSW Microtec. http://www.ksw-microtec.de/www/startseite_de.php

[10] SFA Co., http://www.sfa.co.kr

[11] Blood Center, Laboratory Medicine, Severance Hospital. http://www.severance.or.kr/medical_guide/dept/lab/blood_center/ 